\section{Schlechte Prognose bei hohem IL-10-Spiegel}

Interleukin 10 spielt eine zentrale Rolle im Immunsystem. Aktuelle Untersuchungen weisen darauf hin, dass dieses Zytokin mit der Prognose von Patienten mit hepatozellulärem Karzinom assoziiert ist.

nterleukin 10 (IL-10) ist am virusinduzierten Entzündungsgeschehen in der Leber beteiligt. Zudem hat ein Teil der Patienten mit hepatozellulärem Karzinom erhöhte IL-10-Spiegel. Bei Patienten mit operablen Tumoren wurde beobachtet, dass IL-10-Spiegel über $12 \mathrm{pg} / \mathrm{ml}$ mit einer schlechteren postoperativen Prognose einhergehen. Nun wurde die prognostische Bedeutung des IL10-Spiegels bei Patienten mit inoperablem hepatozellulärem Karzinom untersucht.

Von 222 Patienten mit inoperablem hepatozellulärem Karzinom in der Studie waren $89,1 \%$ Männer, das durchschnittliche Alter lag bei 59,9 Jahren. $64,9 \%$ hatten radiologische Anzeichen einer Zirrhose, 82,4\% ein Hepatitis-BVirus-Oberflächenantigen und 65,8\% einen Lebertumor im Barcelona Clinic Liver Cancer-Stadium C.

Der mittlere log IL-10-Spiegel im Serum betrug 1,1 pg/ml. 146 Patienten hatten IL-10-Spiegel über $1 \mathrm{pg} / \mathrm{ml}$. Diese Gruppe hatte ein signifikant schlechteres Gesamtüberleben als Patienten mit Spiegeln $\leq 1,0 \mathrm{pg} / \mathrm{ml}$ (5,0 vs. 14,9 Monate; Hazard Ratio [HR] 2,192; $\mathrm{p}<0,0001)$. Auch in den multivariaten Analysen nach Berücksichtigung verschiedener Faktoren blieb der IL-10-Spiegel ein unabhängiger Prognosemarker für das Überleben. Darüber hinaus konnte der IL-10-Spiegel innerhalb verschiedener Tumorstadien einen Hinweis auf das Ge- samtüberleben liefern, wobei hohe IL10-Spiegel mit einer schlechteren Prognose assoziiert waren.

Patienten mit hohen IL-10-Spiegeln hatten zudem eine schlechtere Leberfunktion und eine Hepatitis mit stärkerer Aktivität, was sich in höheren HBVDNA- und höheren Alanin-Transaminase-Werten (ALT) niederschlug. Ein erhöhter Serum-IL-10-Spiegel scheint eher die Folge von Leberschäden durch zirrhotische Prozesse zu sein als Folge einer hohen Tumorbelastung.

Fazit: Der Serum-IL-10-Spiegel ist auch bei Patienten mit inoperablem hepatozellulärem Karzinom ein unabhängiger prognostischer Faktor. Das Zytokin könnte die bisher existierenden TumorStaging-Systeme ergänzen.

Judith Neumaier

Chan SL et al. A study of circulating interleukin 10 in prognostication of unresectable hepatocellular carcinoma. Cancer. 2012;118(16):398492.

\title{
Epigenetische Therapie vielversprechend
}

\section{In der Pathogenese des hepatozellulären Karzinoms sind auch epigenetische Veränderungen von Bedeutung. Kann ein Histon-Deacetylase-Inhibitor diesen Prozess beeinflussen?}

Bism ei der Entwicklung eines hepatozellulären Karzinoms spielen zwei epigenetische Prozesse eine Rolle: Die Methylierung der CpG-Inseln in den Promotorregionen einiger Tumorsupressorgene sowie die Histon-Acetylierung, die vom Gleichgewicht der Histon-AcetylTransferasen und -Deacetylasen abhängt. Belinostat ist ein Histon-Deacetylase-Inhibitor, der im hepatozellulären Tumor Apoptose und Regression induzieren kann, aber normale Leberzellen unbeeinflusst lässt.

In dieser Studie wurde erstmals die Wirksamkeit einer epigenetischen Therapie bei Patienten mit fortgeschrittenem, nicht resezierbarem hepatozellulären Karzinom und leichter chronischer Leberfunktionsstörung untersucht. Belinostat wurde an den Tagen 1-5 alle drei Wochen i.v. verabreicht - in Dosen von $600 \mathrm{mg} / \mathrm{m}^{2} / \mathrm{d}$ (Level 1), $900 \mathrm{mg} / \mathrm{m}^{2} / \mathrm{d}$ (Level 2), $1.200 \mathrm{mg} / \mathrm{m}^{2} / \mathrm{d}$ (Level 3) und
$1.400 \mathrm{mg} / \mathrm{m}^{2} / \mathrm{d}$ (Level 4). Ziel der PhaseI-Studie mit 18 Patienten war, dosislimitierende Toxizitäten sowie die maximal verträgliche Dosis zu bestimmen und die Pharmakokinetik zu untersuchen.

Letztere verlief von 600 bis $1.400 \mathrm{mg} /$ $\mathrm{m}^{2}$ linear, ohne dass es zu einer signifikanten Akkumulation gekommen wäre. Die maximal verträgliche Dosis wurde nicht erreicht.

Zusätzlich wurde bei 42 Patienten in einer Phase-II-Studie die Wirksamkeit der höchsten Dosis von $1.400 \mathrm{mg} / \mathrm{m}^{2} \mathrm{Be}$ linostat untersucht. Der primäre Endpunkt war das progressionsfreie Überleben, die wichtigsten sekundären Endpunkte das Ansprechen gemäß RECISTKriterien und das Gesamtüberleben.

Ein partielles Ansprechen erreichten 2,4\% der Patienten, eine Stabilisierung der Erkrankung 45,2\%. Das mediane progressionsfreie Überleben betrug 2,6, das Gesamtüberleben 6,6 Monate.
Weitere Untersuchungen ergaben, dass die Rate für ein partielles Ansprechen oder eine Stabilisierung der Erkrankung bei Tumoren mit hohen HR23B-Histoscores $58 \%$ betrug und bei Tumoren mit niedrigen HR23B-Histoscores $14 \%$; der Unterschied war signifikant $(\mathrm{p}=0,036)$.

Toxizitäten vom Grad $\geq 3$ traten bei mehr als $5 \%$ der Patienten auf, am häufigsten Bauchschmerzen, Hyperbilirubinämie, erhöhte ALT-Werte, Anämie und Erbrechen. Signifikante Neutropenien und Thrombozytopenien wurden nicht beobachtet. Eine leichte Fatigue trat bei mehr als der Hälfte der Patienten auf.

Fazit: Die epigenetische Therapie mit Belinostat führte zu einer Tumorstabilisierung und war im Allgemeinen gut verträglich. Die HR23B-Expression beeinflusste das Ansprechen. Judith Neumaier

Yeo W et al. Epigenetic therapy using belinostat for patients with unresectable hepatocellular carcinoma: A multicenter phase I/II study with biomarker and pharmacokinetic analysis of tumors from patients in the Mayo Phase II Consortium and the Cancer Therapeutics Research Group. J Clin Oncol. 2012;30(27):3361-7. 